\title{
Improved Ensemble Empirical Mode Decomposition and Its Application
}

\author{
Xuze Lin ${ }^{12}$ \\ 5th Dep., The Second Artillery Engineering University, Group 4504, No.2 Tongxin Road, Hongqing Town, \\ Baqiao District, Xi'an City, Shaanxi Province,710025, China \\ E-mail: 280080322 @qq. com
}

\section{Yanping $\mathrm{Cai}^{3}$}

5th Dep., The Second Artillery Engineering University, Group 4504, No.2 Tongxin Road, Hongqing Town, Baqiao District, Xi'an City, Shaanxi Province,710025, China

E-mail:caiyanping502@163.com

\section{Xinjun Wang}

5th Dep., The Second Artillery Engineering University, Group 4504, No.2 Tongxin Road, Hongqing Town, Baqiao District, Xi'an City, Shaanxi Province,710025, China

E-mail:wangxinjun502@163.com

\section{Fang Wang}

5th Dep., The Second Artillery Engineering University, Group 4504, No.2 Tongxin Road, Hongqing Town, Baqiao District, Xi'an City, Shaanxi Province,710025, China

E-mail: wang502fange163. com

\begin{abstract}
A white noise parameter and a number of ensembles for EEMD adaptively obtained method (Adaptive Ensemble Empirical Mode Decomposition, AEEMD) is proposed to tackle the problem that the EEMD parameters are inaccurately chosen by the people's experience. First of all, the energy of the added noise is calculated according to SNR (Signal to Noise Ratio) and thus the amplitude parameter of the original signalscan be obtained. Then the number of ensembles is calculated according to the amplitude parameter and the expected relative error of decomposition. Simulations prove that AEEMD is more effective than EMD and EEMD in decomposition, and AEEMD can obtain the two parameters adaptively. The last but not the least, AEEMD is applied to the fault diagnosis of rolling bearings in combination with the envelope spectrum analysis. The result indicates that the simulated bearing fault features as extracted by AEEMD can be used as the basis of the mechanical fault diagnosis.
\end{abstract}

CENet2015

12-13 September 2015

Shanghai, China

\section{${ }^{1}$ Speaker \\ ${ }^{2}$ Correspongding Author}

${ }^{3}$ The research in this paper was supported by Natural Science Basic Research Plan of Shaanxi Province, China (Program NO.: 2013JQ8023) and National Natural Science Foundation of China (Program NO.:51405498) (Program NO.: 61201449). 


\section{Introduction}

The traditional signal analysis method based on Fourier transform is less effective at the moment of processing the nonlinear and non-stationary signals. To solve the problem, the wavelet transform (WT) has been proposed by some scholars; however, the wavelet kernel functions and the decomposition level need to be selected artificially in WT. Once the functions and the level are selected, the decomposition scale is fixed, which makes the decomposition unsatisfactory. The empirical mode decomposition (EMD)[1] can adaptively generate a collection of intrinsic mode functions (IMF), which have different scales to solve the timefrequency analysis problem of nonlinear and non-stationary signal; but there are still problems such as mode aliasing[2]. In order to solve this problem, Huang and Wu proposed the ensemble empirical mode decomposition (EEMD)[3], which can eliminate the intermittent phenomenon in the original signals and make the signal continuity of different scales by using the statistical characteristics of Gaussian white noise with the uniform distribution of frequency so as to effectively eliminate the mode aliasing. The EEMD has been widely used[4,5,6], but EEMD may not be able to completely eliminate the modal aliasing. There are two main reasons. Y. T. Zhou considered EEMD stopping criteria would be influenced by the mean square error of signals to be decomposed and the screening would not be completely in the process of filtering, which would lead low-frequency part of Gaussian white noise mixed in the high-frequency IMF. It makes EEMD insignificant in the low frequency part. He further put forward decomposing Gaussian white noise with EMD method and used the sum of minimum absolute value of decomposition results as stopping threshold. With improvement of the stopping criterion, the accuracy of EEMD would be improved[7]. Another reason is that amplitude of the added noise and number of ensembles for EEMD should be determined in dependence on experience. If the parameters are determined improperly, the decomposition effect would be worse than EMD; at the same time the method could undermine its adaptability.

In order to solve these problems, an improved EEMD - adaptive ensemble empirical mode decomposition (AEEMD) is presented. Firstly, the method adaptively calculates the standard deviation of amplitude of added white noise by the original signal with noise under suitable SNR (Signal to Noise Ratio); then, the amplitude of added noise and number of ensembles for EEMD are determined. The AEEMD can not only effectively eliminate the mode aliasing, but also make the parameters adaptive; in this sense, it is significant.

\section{Principle of EEMD Method}

In order to solve the mode aliasing of EMD, Huang and $\mathrm{Wu}$ proposed the ensemble empirical mode decomposition method (EEMD). In case of decomposition, the white Gaussian noise would be added to the original signal to effectively eliminate the mode aliasing of traditional empirical mode decomposition. In principle, EEMD is to use the statistical characteristics of Gaussian white noise with the uniform distribution of frequency so that the signal components can be automatically mapped to the scale plane related to the background white noise and make the signal continuity of different scales. In order to eliminate the interference caused by each addition of white noise, the signal with noise is decomposed for many times and each intrinsic mode functions (IMF) are taken on average; as a result, the interference becomes small. The results of EEMD are approximate IMF. EEMD steps are reviewed as follows:

(1) add random Gaussian white noise sequence to the signal to be decomposed as

$$
x_{M}(t)=x(t)+k \cdot n_{M}(t)
$$

In the formula, $x_{M}(t)$ and $n_{M}(t)$ represent the signals with noise to be decomposed for $M$-st times and the corresponding white noise as added; $k$ represents the amplitude parameter of white noise. 
(2)Use EMD to decompose the $M$-st signal with noise into a collection of IMFs and repeat Step (2.2) for $\mathrm{N}$ times.

(3)Calculate the average of each IMF after N times' decomposition as

$$
i m f_{i}=\frac{1}{N} \sum_{j=1}^{N} i m f_{i, j}
$$

In which $i=1,2, \cdots, I, j=1,2, \cdots, N$.

EEMD can solve the modal aliasing problems effectively by using the white noise assisted method, but there's a problem that the decomposition effect depends on $k$, white noise amplitude, and $N$, number of ensembles for EEMD. At present, in the applications of EEMD, people generally choose $N=100 \sim 200$ and $k=0.15 \sim 0.25[8,9,10]$. The method is too empirical and does not change with characteristics of the signals to be decomposed. It will destroy the adaptability of EEMD. Y. P. Cai, L. Chen and X. He, et al. have established the white noise adding criterion for EEMD, and thus put forward the method to adaptively obtain the amplitude in respect of the added noise and number of ensembles for EEMD by analyzing the existing problems in EEMD method[11,12,13]. The method applies the ratio of the amplitude standard deviation of high-frequency component and low-frequency component of signal to determine $k$ and $N$ by setting the expected decomposition error. This method has certain application value but it is not easy to distinguish the high-frequency components from low-frequency components of signals in the process of calculation; at the same time, large number of ensembles for EEMD makes the decomposition process to be slow, which fails to satisfy the timeliness of fault diagnosis.

\section{AEEMD Method}

EEMD is a noise-assisted signal processing method, of which the decomposition effect depends on the choice of noise amplitude and number of ensembles for EEMD to a large extent. $\mathrm{Z}$. $\mathrm{H}$. $\mathrm{Wu}$ et al. have studied the relations between white noise amplitude parameter and the number of ensembles for EEMD[14]. Studies showed the relations among $N$, number of ensemble, $k$, white noise amplitude parameter, and $e$, the impact to decomposition results. The relationship is shown as follows:

$$
e=k / \sqrt{N}
$$

where $N$ is the number of ensemble for EEMD; $k$ is the ratio of amplitude standard deviation of the added white noise and the original signal; $e$ is the minimum of expected relative decomposition error, that is, the relative error between the original signal and the sum of each IMF.

SNR is of practical significance, which reflects the interference degree of the noise to the original signal. When the noise assisted analysis method is needed, the SNR is generally lower than $40 \mathrm{~dB}$; but it can not be too low or the original signal will submerge in the noise. EEMD actually is a noise assisted analysis method. Different amplitudes of the white noise, which is added to the EEMD, actually determine different SNRs of the signal. Based on the relationship above, the method using SNR of the signal to determine the white noise amplitude parameter is proposed. The formula of SNR is shown as follows:

$$
S N R=10 \log _{10}\left(p_{s} / p_{n}\right)
$$

In which, $p_{s}$ is the energy of the signal and $p_{n}$ is the noise energy. Once the energy of the original signal is determined, the energy of the added white noise can also be determined; then the amplitude standard deviation of the white noise can be obtained as well. Now, what to be determined is how much of a SNR can make a better EEMD result. Several tests show that when SNR takes 15 to $20 \mathrm{~dB}$, EEMD is faster, the decomposition result is more accurate and the performance to resist the mode aliasing is better. The flowchart of improved EEMD is shown in Fig. 1: 


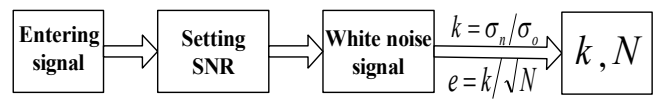

Figure 1 : Flowchart of AEEMD

\section{Simulation Test}

In order to demonstrate the validity of AEEMD, we form the simulated signal. The simulated signal is the superposition of three sinusoidal signals of different frequencies. Firstly, we use EMD to decompose the signal with the result shown in Fig. 2. We can see that the first IMF is on behalf of high-frequency component; the third IMF is a stationary component. High frequency components and low frequency components mix together and can not be separated in the second IMF, which is the mode aliasing.

Then we use EEMD to decompose the signal by setting $k=0.2, N=200$ according to the experience with the result shown in Fig. 3. It can be found that the three kinds of frequency components are separated, but there is still the mode aliasing in the first and the second IMF. The amplitude parameter of white noise and the number of ensembles for EEMD can't match the signal to be decomposed.

Next, we use AEEMD to decompose the signal by setting $e=1 \%, \mathrm{SNR}=17 \mathrm{~dB}$. The decomposition result is shown in Fig. 4. Compared with Fig. 3, we can find that the three components can be separated untied both by EEMD and AEEMD, but AEEMD for decomposing is more accurate and faster than EEMD. In addition, AEEMD can also eliminate the mode aliasing and better reflect the essence of the original signal.

Table 1 shows the performance of EMD, EEMD and the improved EEMD as proposed in Literature $[2,6,14]$ and AEEMD as proposed in this paper. It can be seen that not only the decomposition time of improved EEMD in this paper is short, but also the decomposition results are very accurate; therefore, the AEEMD as proposed by us is effective.

\begin{tabular}{ccccc}
\hline Method & $\mathrm{k}$ & $\mathrm{N}$ & Time consuming (s) Decomposition effect \\
\hline EMD & $\backslash$ & $\backslash$ & 0.12 & Very bad \\
EEMD & 0.2 & 200 & 29.16 & Not accurate \\
Improved EEMD of others & 0.15 & 220 & 33.51 & Accurate \\
Improved EEMD in this paper & 0.14 & 199 & 23.63 & Accurate \\
\hline
\end{tabular}

Table 1: Comparison of Decomposition Results of Four Methods

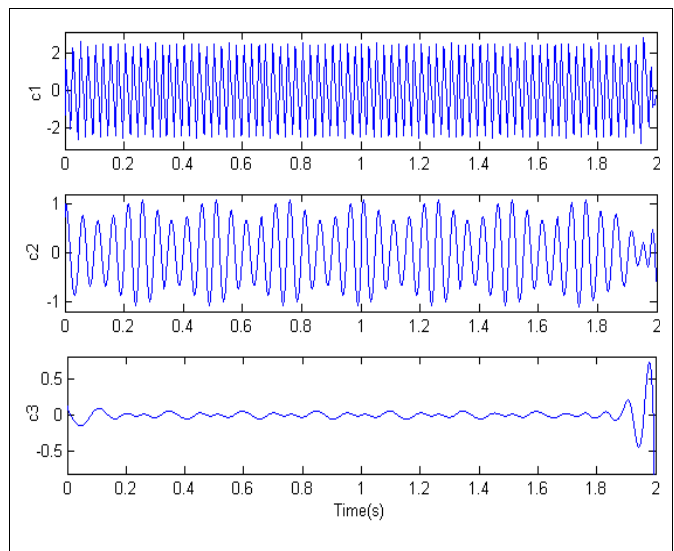

Figure 2: EMD of Simulated Signal

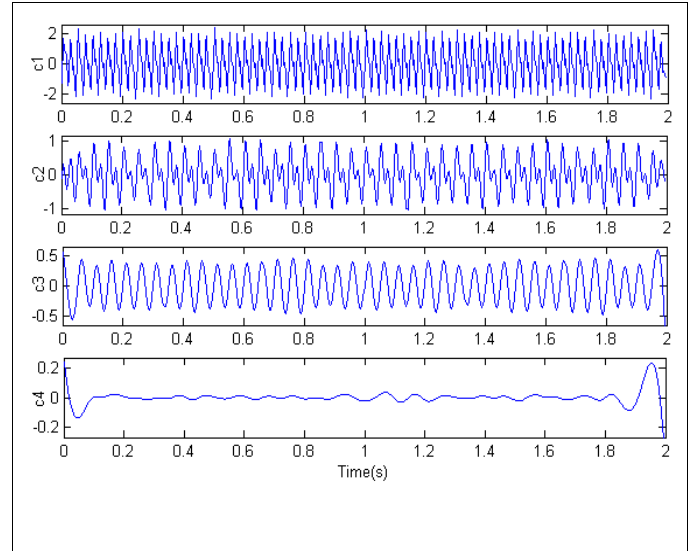

Figure 3:EEMD of Simulated Signal 


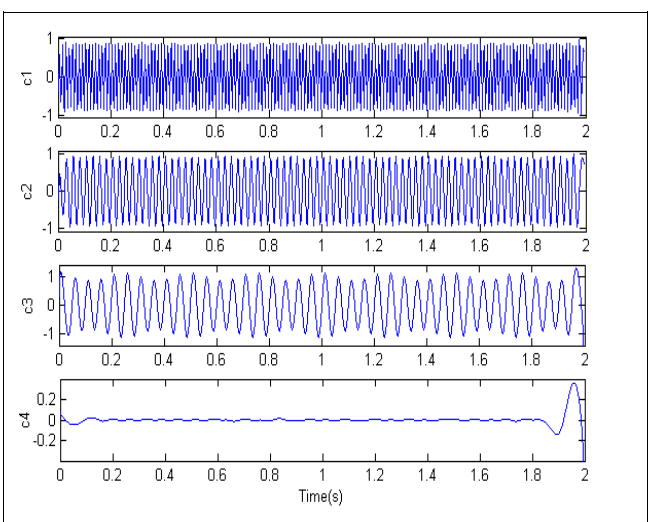

Figure 3:AEEMD of Simulated Signal

\section{Applications}

When the ball bearing outer ring fails, the vibration signal should show up as a periodic continuous impulse. We use MATLAB programming to randomly generate a set of impulse responses of outer ring partial failure as the outer ring fault signal model. The failure frequency is $200 \mathrm{~Hz}$; the sampling frequency is $32768 \mathrm{~Hz}$ and the resonant frequency is $9000 \mathrm{~Hz}$. In order to characterize the early weak fault signal, the outer ring fault signal is mixed with the Gaussian white noise with certain energy and the same length as the original fault signal.

Firstly, we use EMD to decompose the fault signal with the result shown in Fig. 5. There are varied degrees of mode aliasing from the second to the sixth IMF. Then AEEMD is used instead of EMD, adaptively obtaining white noise parameters of which $e$ is 0.1412 and $N$ is 199 with the result shown in Fig. 6. In Fig. 6, the mode aliasing is completely eliminated and the white noise parameters are adaptively obtained. Its decomposition result is more accurate and more detailed to reflect period characteristics and impact characteristics of bearing fault signal so that it can contribute to the subsequent fault types recognition.

The envelope spectrum of the first three IMFs of AEEMD is analyzed with the results shown in Fig. 7. The failure frequency is $208 \mathrm{~Hz}$ actually (theoretically $200 \mathrm{~Hz}$ ) and the frequency doubling line $(400 \mathrm{~Hz})$ is shown clearly in the envelope spectrum; therefore, the proposed AEEMD can be effectively applied to the fault diagnosis of rolling bearings in combination with the envelope spectrum analysis.

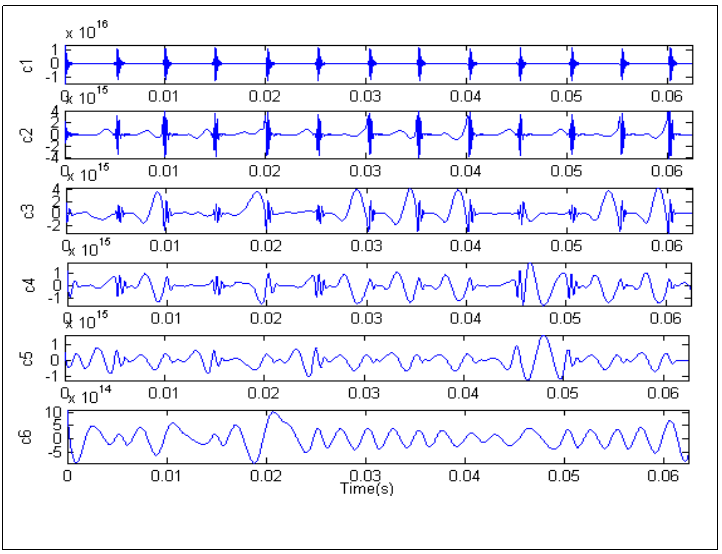

Figure 5 : EMD of Outer Ring Fault Signal 


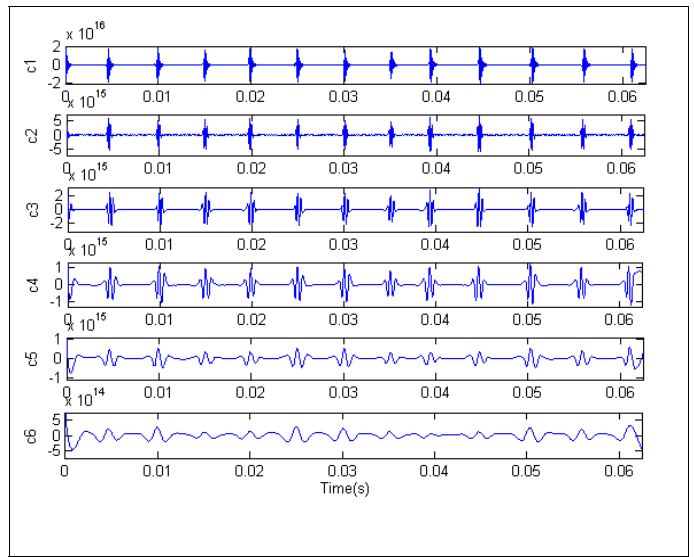

Figure 6: AEEMD of Outer Ring Fault Signal

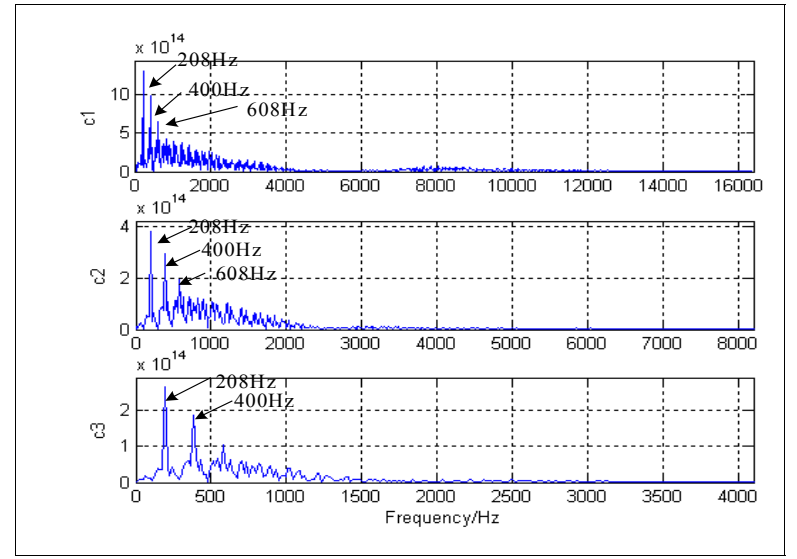

Figure7: Envelope Spectrum of IMF1 IMF3

\section{Conclusion}

Based on the research of EEMD principle, it is pointed out that one of the defects of EEMD is the selection of parameters. Then we analyze the effects of different intensities of the white noise based on the results of EEMD. On this basis, we propose using SNR to determine the white noise parameters in EEMD. Studies have shown that: when the SNR takes 15 to 20dB, EEMD is faster and the decomposition result is more accurate and the performance to resist mode aliasing is better. Simulation and experimental studies show the effectiveness on resisting mode aliasing of the improved EEMD and the adaptability to obtain the white noise parameters. It has proved that the proposed AEEMD can be effectively applied to the fault diagnosis of rolling bearings in combination with the envelope spectrum analysis.

\section{References}

[1] N. E. Huang, Z. Shen, S. R. Long, M. C. Wu, H. H. Shih, Q. A. Zheng. The empirical mode decomposition and the Hilbert spectrum for nonlinear and non-stationary time series analysis[J]. Proceedings of the Royal Society A: Mathematical, Physical and Engineering Sciences. 454(1971): 903-995(1998)

[2] C. Zhang, J. J. Chen. Contrast of ensemble empirical mode decomposition and empirical mode decomposition in mode mixture[J]. Journal of Vibration and Shock. 29(S): 87-90 (2010)

[3] Z. H. Wu,N. E. Huang. Ensemble empirical mode decomposition: a noise assisted data analysis method[J]. Advances in Adaptive Data Analysis. 1(1): 1-41(2009) 
[4] R. X. Chen, B. P. Tang, J. H. Ma. Adaptive de-noising method based on ensemble empirical mode decomposition for vibration signal[J]. Journal of Vibration and Shock. 31(15): 82-86(2012)

[5] L. L. Gao, X. Q. Wang, W. F. Jiang. EEMD denoising arithmetic of unsteady vibration signals of diesel engine [J]. Coal Mine Machinery. 33(9): 278-280(2012)

[6] Y. Zhang, W. Tian, S. P. Liu. Fire time series forecasting based on ensemble empirical mode decomposition[J]. Computer Engineering. 38(24): 152-155 (2012)

[7] Y. T. Zhou, S. Q. Zhou, Y. H. Yao. Improved ensemble empirical mode decomposition to reduce modal aliasing $[\mathrm{J}]$. Journal of Chongqing University of Technology (Natural Science). 29(1): 111$114,130(2015)$

[8] G. Y. Hou, Y. Lv, H. Xiao, T. Qin. Application of adaptive morphology in gear fault diagnosis based on EEMD[J]. Journal of Vibration and Shock. 33(18): 145-148 (2014)

[9] C. L. Li, F. R. Kong, W. G. Huang, H. Chen, C. Wang, Z. Z. Yuan. Rolling bearing fault diagnosis based on EEMD and Laplace wavelet[J]. Journal of Vibration and Shock. 33(3): 63-69, $88(2014)$

[10]X. T. Wu, M. Yang, X. H. Yuan, T. K. Gong. Bearing fault diagnosis using EEMD and improved morphological filtering method based on kurtosis criterion [J]. Journal of Vibration and Shock. 34(2): 38-44(2015)

[11] Y. P. Cai, A. H. Li, B. Xu, P. Xu, Y. P. He. Adaptive guideline of ensemble empirical mode decomposition with Gauss white noise[J]. Journal of Vibration, Measurement \& Diagnosis. 31(6): 709-714, 811(2011)

[12]L. Chen, G. S. Tang, Y. Y. Zi, Z. N. Feng, K. Li. Application of adaptive ensemble empirical mode decomposition method to electrocardiogram signal processing $[\mathrm{J}]$. Journal of Data Acquisition \& Processing. 26(3): 361-366(2011)

[13]X. He, H. L. Wang, W. Jiang, L. Wang. Improved adaptive EEMD method and its application[J]. Journal of System Simulatio. 26(4): 869-873(2014)

[14]Z. H. Wu, N. E. Huang. A study of the characteristics of white noise using the empirical mode decomposition method[J]. Proceedings Royal Society of Lond. 460(2046): 1597-1611(2004) 